\title{
Measured WEB Performance in GPRS, EDGE, UMTS and HSDPA with and without Caching
}

\author{
Philipp Svoboda \\ Institute of Communications and \\ Radio-Frequency Engineering \\ Vienna University of Technology \\ Gusshausstrasse 25/389, A-1040 \\ psvoboda@nt.tuwien.ac.at
}

\author{
Fabio Ricciato \\ ftw. Forschungszentrum \\ Telekommunikation \\ Donau-City-Strasse 1, \\ A-1220 Vienna, Austria \\ ricciato@ftw.at
}

\author{
Werner Keim, Markus Rupp \\ Institute of Communications and \\ Radio-Frequency Engineering \\ Vienna University of Technology \\ Gusshausstrasse 25/389, A-1040 \\ \{wkeim,mrupp\}@nt.tuwien.ac.at
}

\begin{abstract}
In this paper we present results from a measurement campaign evaluating the web performance of different mobile Internet access technologies with and without caching. We measured in GPRS, EDGE, UMTS and HSDPA radio access networks of a mobile operator in Austria. The operator has implemented a web-proxy for performance improvement. To evaluate the impact on the different technologies we ran the setup with and without the web-proxy. We defined the download time of web pages as the performance index for our work. We developed a tool to capture this parameter and derived the index for a set of web sites frequently used by the customers in the network. Beginning with an overall comparison we will present detailed analysis for special cases. We evaluated the impact of the proxy system to different site configurations using the performance index. Finally we compared the performance numbers for HTTP with results collected from FTP transfers.
\end{abstract}

\section{Introduction}

The fast growth of the Internet has also influenced the evolution of mobile networks. The introduction of GPRS (General Packet Radio Service) to the mobile market was a step to cover the growing demand for packet switched services. This was the first move from the pure voice oriented GSM (Global System for Mobile Communications) network to a system supporting a wide range of new services. With a throughput of $\approx 80 \mathrm{kbit} / \mathrm{s}$ and a RTT (RoundTrip Time) of $\approx 500 \mathrm{~ms}$ GPRS was not competitive to wired Internet access. The introduction of UMTS (Universal Mobile Telecommunications System) changed this fact. The available bandwidth increased to $384 \mathrm{kbit} / \mathrm{s}$ and the RTT dropped to $\approx 140 \mathrm{~ms}$. This performance gain in combi-
Table 1. Measured ping times (32 bytes)

\begin{tabular}{|l|r|r|r|}
\hline Technology & Bandwidth (down/up) & Mean & Std \\
\hline GPRS & $80 / 40 \mathrm{kbit} / \mathrm{s}$ & $488 \mathrm{~ms}$ & $146 \mathrm{~ms}$ \\
EDGE & $240 / 120 \mathrm{kbit} / \mathrm{s}$ & $504 \mathrm{~ms}$ & $89 \mathrm{~ms}$ \\
UMTS & $384 / 128 \mathrm{kbit} / \mathrm{s}$ & $142 \mathrm{~ms}$ & $58 \mathrm{~ms}$ \\
HSDPA & $1800 / 384 \mathrm{kbit} / \mathrm{s}$ & $91 \mathrm{~ms}$ & $43 \mathrm{~ms}$ \\
ADSL & $1000 / 256 \mathrm{kbit} / \mathrm{s}$ & $10.9 \mathrm{~ms}$ & $0.8 \mathrm{~ms}$ \\
\hline
\end{tabular}

nation with flat rate contracts has introduced mobile Internet access to a large population. Complete UMTS coverage is expensive for the operator to gain. Therefore, in order to save costs, some of the operators migrated their legacy GPRS equipment to EDGE (Enhanced Data rates for GSM Evolution), which provides three times the bandwidth of GPRS. Note that the RTT in this case still remains at the level of GPRS, see Table 1. HSDPA (High Speed Downlink Packet Access), an evolution of UMTS, offers more than $1.8 \mathrm{Mbit} / \mathrm{s}$ at a very low delay of $\approx 90 \mathrm{~ms}$. The RTT values we used in this paragraph are well known in literature, for example see [1]. However, we also measured these values using ping requests, contacting the $G_{i}$ interface of the GGSN. The results for 100 ping packets with a payload size of 32 bytes is shown in Table 1 .

As we have shown in [2] the IP traffic in the network of a mobile operator, in terms of volume, is dominated by HTTP traffic, measured in bytes over TCP port 80 . The intention of this paper is to find out how the different access technologies with their different parameters perform to the perspective of a web user.

The rest of this paper has been organized in the following way. In Section 2 we show the measurement setup, including a flowchart for the coded software and a description of the location and hardware involved in the measurement. Section 3 presents the overall results for all technologies with and without a proxy. In Section 4 we discuss the detailed results for special pages and compare the HTTP num- 


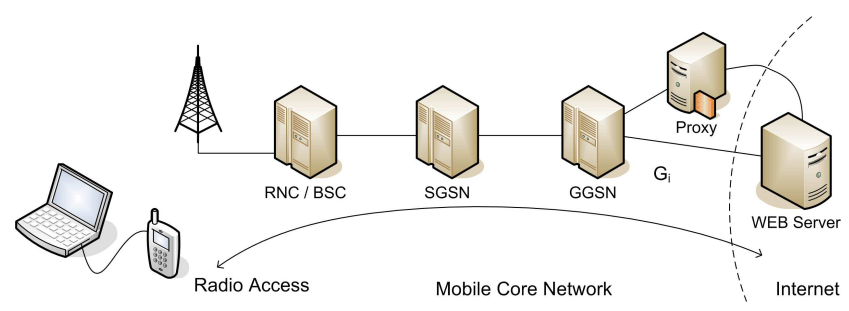

Figure 1. Basic measurement setup

bers to FTP measurements. Related work is cited in Section 5. The conclusion is drawn in Section 6.

\section{Measurement Setup}

We conjectured that the web-user's satisfaction is based upon the time he is waiting for a page download to finish. Therefore, we used the download time of web-pages as the performance indicator for the different technologies. We selected eleven live sites with a high popularity according to the log file of the proxy system implemented by the mobile network operator. Web-proxy systems are often used by mobile operators to improve the service performance. They can be placed at the $G_{i}$ interface, that connects the mobile gateway with the Internet. These programs often also include special optimizations for mobile networks.

In order to obtain the performance of HTTP we developed a tool capable of scripted web page downloads. Fig. 1 shows the principle measurement setup. The web servers were not located within the operator's network, however the chosen sites were all hosted in Austria. Therefore, we assumed a minor impact due to the Internet access of the operator.

\subsection{The tool: IE_Control}

In [2] it is shown that in a mobile $3 \mathrm{G}$ network the large majority of web users accesses the network with datacards from a PC. Customers browsing with a handset mainly go for WAP portals. Therefore, we decided to use PC specific web browsers. In [3] it states that $\geq 85 \%$ of all web users still stick to the Internet Explorer (IE) of Microsoft. Therefore, we used a Visual C++ v6 environment to derive an IE class ${ }^{1}$. The tool derives an IE-ActiveX Control, implements it in a MFC environment and measures the time between start and end of a page download. Web pages are downloaded according to an input file. Furthermore, a dial up management was added to automatize the measurement. Additional output obtained by IE-function, e.g. volume, time stamps and number of objects, is written into a log file. A principle flowchart is shown in Fig. 2. We decided to make the tool publicly available ${ }^{2}$.

\footnotetext{
${ }^{1}$ Preliminary results obtained with a Mozilla control lay within 5\%.

${ }^{2}$ www.nt.tuwien.ac.at/about-us/staff/ philipp-svoboda/
}

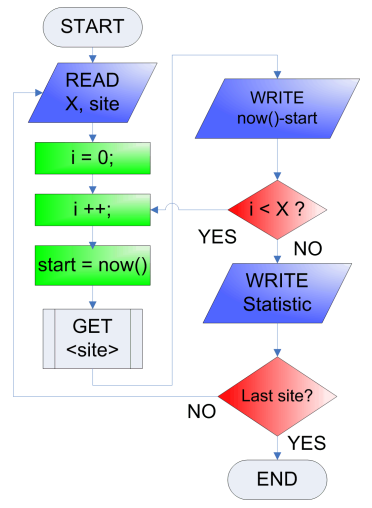

Figure 2. Flowchart of IE_Control

\subsection{The hardware}

The used hardware was an OPTION HSDPA card model $3 \mathrm{G}+$ EMEA. The modem is capable of GPRS, EDGE, UMTS and HSDPA access. The location itself was chosen in a way that it had no influence to the measurement results. We used a standard notebook running the IE_Control tool recording the data sets. The operating system was Windows XP with standard TCP settings.

\subsection{The measurement}

The datasets were recorded during the night of the 2526th of February 2006. Let us first consider the UMTS case, as it is the simplest scenario. In UMTS every user is assigned to a DCH (see [4, p. 82]), therefore each experiment has the same dedicated bandwidth. However, in case of inactivity the radio access network forces the user to a FACH (Forward Access Channel) in order to save resources. We set the time between two consecutive downloads in order to keep the DCH open.

In GPRS, EDGE and HSDPA the channel is shared and other active users will impact the measurement results. To evade this problem we measured during night hours. The location was an office building of the mobile operator featuring an indoor micro cell coverage. Each site was downloaded 100 times in each access technology for the proxy and the non-proxy case. Prior to the recorded downloads the site was transferred one time to setup the radio channel. Non-proxy measurements used a special APN (Access Point Name) bypassing the proxy system. In this scenario persistent TCP connections will be reused for consecutive downloads. We selected our sites from the top 20 of the caching report of the mobile operator. Table 2 shows a detailed list of the eleven sites used. We extracted the parameters HTTP (gzip) and HTTP (pipe) , they indicate the use of compression respectively pipelining at the server. The last column indicates the number of off site advertisement links. Such links need an additional TCP connection and will slow down the transfer. In order to compare the results with fixed 
Table 2. Sites in the test sample

\begin{tabular}{|l|r|r|l|l|l|}
\hline Site & \multicolumn{2}{|c|}{ Size } & \multicolumn{2}{c|}{ HTTP } & Adv. \\
& gzip & normal & gzip & pipe & \\
\hline amazon.at & $337 \mathrm{kB}$ & $338 \mathrm{kB}$ & YES & YES & 3 \\
aon.at & $328 \mathrm{kB}$ & $397 \mathrm{kB}$ & NO & YES & 2 \\
ba-ca.com & $28.1 \mathrm{kB}$ & $31.2 \mathrm{kB}$ & NO & YES & - \\
ebay.at & $168 \mathrm{kB}$ & $167 \mathrm{kB}$ & YES & NO & - \\
gmx.at & $220 \mathrm{kB}$ & $227 \mathrm{kB}$ & YES & NO & - \\
google.at & $14.9 \mathrm{kB}$ & $14.9 \mathrm{kB}$ & YES & YES & - \\
heise.de & $75.0 \mathrm{kB}$ & $74.8 \mathrm{kB}$ & YES & NO & - \\
love.at & $175 \mathrm{kB}$ & $175 \mathrm{kB}$ & YES & YES & 3 \\
orf.at & $110 \mathrm{kB}$ & $138 \mathrm{kB}$ & NO & YES & 2 \\
sms.at & $220 \mathrm{kB}$ & $226 \mathrm{kB}$ & NO & NO & - \\
websingles.at & $372 \mathrm{kB}$ & $451 \mathrm{kB}$ & NO & NO & - \\
\hline
\end{tabular}

wired Internet accesses we reran the measurement using a standard ADSL line (down: 1Mbit/s; up: 256kbit/s).

\subsection{The basic functions of the proxy}

The proxy system used provides the following features: caching, HTTP conversion and compression in real time. At first, the proxy system acts as a normal web caching system. This feature reduces the used bandwidth on $G_{i}$ and the response time to user requests. The compression reduces the size of HTML text objects by applying the gzip algorithm on the document. The HTTP conversion option of the proxy system is a manipulation of the connection management. The proxy merges all objects for one HTTP site into a single TCP stream. To support all clients, the proxy uses HTTP 1.0 connections with a "keep alive" entry in the header. Users have only one TCP slow start per page.

\section{Results}

Table 3 holds the relative increase of download time $(d t)$ and the resulting performance index $\left(P I_{A D S L}\right)$ for different technologies compared to an 1Mbit ADSL access. The values in Table 3 are the medians of the data sets recorded, normalized by ADSL download time $\left(d t_{A D S L}\right)$ presented in column two. $P I_{A D S L}$ (Performance Index compared to ADSL) is calculated according to (1).

$$
P I_{A D S L}=\frac{1}{k-1} \sum_{\text {site }=1}^{k} \frac{\text { median }\left(d t_{\text {site }, \text { Tech } X}\right)}{\text { median }\left(d t_{\text {site }, A D S L}\right)}
$$

HSDPA is often close to ADSL, but does not surpass it in any case. The increase between HSDPA and UMTS is $\geq$ $100 \%$, indicating the huge performance gain offered by the newer technology. In EDGE the proxy system achieves the strongest improvement, bringing it close to UMTS performance. Without the proxy EDGE scores similar to GPRS $\mathrm{p}$, GPRS with proxy. The GPRS values show that this technology is limited by design and it ends up last, with $11-17$ times lower performance than ADSL. Overall the proxy introduces $10-45 \%$ improvement. Systems with a smaller delay achieve smaller gains. EDGE benifits most from the optimizations by the proxy. If we compare Table 3 and 2 we can figure out the gain according to the different optimizations. The next section will show that compression achieves $10-25 \%$ improvement, while the keep-alive feature gains $20-40 \%$.

\section{Discussion of the results}

In this section we will focus on five different web sites, namely: amazon.at, google.at, orf.at, ebay.at and sms.at. The sites include all four combinations of $\mathrm{HTTP}_{\text {(pipe) }}$ (keep-alive used) and $\mathrm{HTTP}_{\text {(gzip) }}$ (compression used) and a mixed scenario. google.at represents the class of highly optimized web sites using both settings. The other extreme is sms.at, it uses no compression and no keep-alive. Compression is also not supported by orf.at however, this site does support keep-alive. The last combination with no keep-alive and compression is covered by ebay.at A mixed scenario is shown in amazon.at. Although this site supports both improvements, it consists of several parts transfered from different servers, mainly due to banner advertisement.

\subsection{Mixed page styles}

Fig. 3 shows the amazon.at boxplot for all technologies with and without proxy. The variance of the results drops with the increasing speed of the connection. Starting on the left side we see that HSDPAp, is nearly as fast as the wired line Internet connection. The proxy optimizes the off-site links and can therefore gain up to $50 \%$. This indicates that for HTTP traffic, HSDPA is a good equivalent to standard Internet connections.

There is a visible gain introduced by the proxy within the technologies. The ranking of the technologies is as expected. However the gap between UMTS and HSDPA is much smaller than the difference in bandwidth, which is approximately 4.7. A factor of approximately two, is more related to the delay reduction in the order of 1.8 (see Table 1). The EDGE $\mathrm{p}$ results are interesting. Here we see a huge gain compared to the standard EDGE scenario, nearly reaching the uncached UMTS values. EDGE has high bandwidth (comparable to UMTS) and high delay (similar to GPRS), therefore the proxy system can gain a lot by reducing the delay. There is also a high gain in the GPRS case. In the uncached GPRS case we discern a high variation, which is due to the shared channel with small resources. In other words the download timespan in GPRS is much larger and therefore the probability of an interruption is higher. The gain in this scenario is $13-35 \%$ increasing from HSDPA to GPRS. 
Table 3. Results for different technologies

\begin{tabular}{|c|c|c|c|c|c|c|c|c|c|c|}
\hline Site & ADSL [sec] & ADSL & HSDPA $_{p}$ & HSDPA & UMTS $_{p}$ & UMTS & EDGE $_{p}$ & EDGE & GPRS $_{p}$ & GPRS \\
\hline amazon.at & $\overline{4.66}$ & $\overline{1.00}$ & $\overline{\overline{1.41}}$ & 2.01 & 2.94 & 3.38 & $\overline{5.26}$ & 8.14 & 8.87 & 12.58 \\
\hline aon.at & 2.73 & 1.00 & 2.06 & 2.38 & 3.06 & 4.18 & 6.62 & 14.13 & 20.47 & 25.96 \\
\hline ba-ca.at & 0.732 & 1.00 & 2.41 & 2.56 & 3.94 & 3.63 & 4.36 & 6.13 & 7.37 & 8.66 \\
\hline ebay.at & 2.59 & 1.00 & 2.64 & 3.16 & 3.35 & 4.38 & 6.41 & 8.02 & 11.30 & 16.02 \\
\hline gmx.at & 3.39 & 1.00 & 1.47 & 1.81 & 5.18 & 6.08 & 4.95 & 13.66 & 10.06 & 21.98 \\
\hline google.at & 0.25 & 1.00 & 2.50 & 2.72 & 5.33 & 5.41 & 7.94 & 8.82 & 14.16 & 15.80 \\
\hline heise.at & 1.17 & 1.00 & 1.75 & 2.28 & 4.09 & 6.26 & 5.79 & 9.73 & 10.34 & 15.99 \\
\hline love.de & 1.52 & 1.00 & 2.12 & 2.50 & 3.86 & 4.36 & 6.84 & 10.02 & 15.90 & 18.87 \\
\hline orf.at & 2.65 & 1.00 & 1.73 & 2.02 & 3.08 & 3.20 & 4.23 & 4.97 & 6.19 & 8.60 \\
\hline sms.at & 3.17 & 1.00 & 1.40 & 1.86 & 3.58 & 4.65 & 5.81 & 7.97 & 10.25 & 18.30 \\
\hline websingles.at & 5.91 & 1.00 & 2.17 & 2.57 & 3.24 & 3.75 & 5.32 & 11.78 & 17.31 & 19.39 \\
\hline $\mathbf{P I}_{\text {ADSL }}$ & - & 1.00 & 1.97 & 2.25 & 3.99 & 4.45 & 5.77 & 10.57 & 11.09 & 16.84 \\
\hline
\end{tabular}

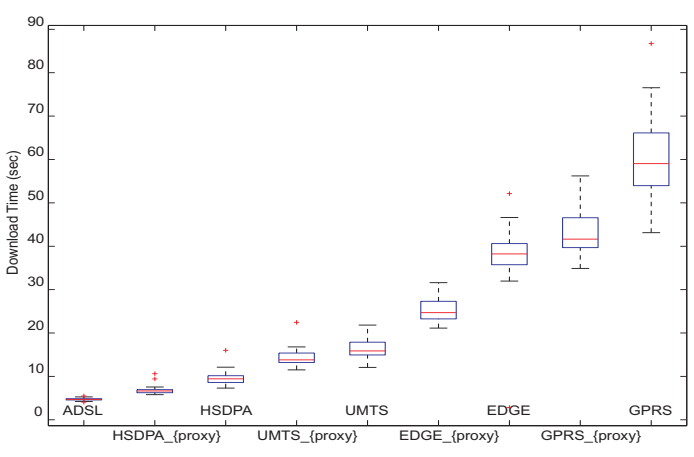

Figure 3. Technology comparison for amazon.at (Box-Jenkins Plot)

HTTP-pipe: YES, HTTP-gzip: YES In Fig. 4(a) the results for google.at are picted. The site is highly optimized and very small. Therefore, we expect two effects, first the gain by the proxy should be small and second the variation of the technologies will be large. The first assumption is met by all technologies HSDPA $(\approx 8 \%)$ and UMTS $(\approx 1 \%)$, EDGE $(\approx 10 \%)$ and GPRS $(\approx 10 \%)$. And also the variation proves to have a similar high level for the different technologies.

HTTP-pipe: YES, HTTP-gzip: NO The boxplot in Fig. 4(b) shows the results for orf.at. This site uses keep-alive, but no gzip compression. The compression rate is approximately 20\% (see Table 2). The gain for fast technologies (HSDPA and UMTS) is smaller than this compression factor, here the system gains $14 \%$ respectively $6 \%$. In this case we conjecture that the main part of the download time is generated from delays caused by the HTTP protocol (GET, ...) and the real data transfer has only a small impact. EDGE and GPRS show a stronger improvement when using the proxy system. The gain is in the order of the compression

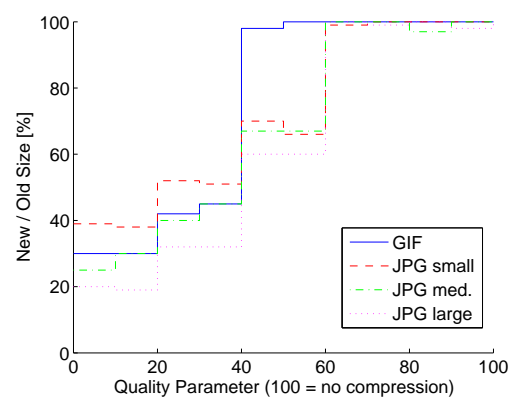

Figure 5. Effectiveness of lossy compression

ratio (EDGE: 15\%, GPRS: 28\%). GPRS, with its low bandwidth, profits most from the reduced page size.

HTTP-pipe: NO, HTTP-gzip: YES Fig. 4(c) displays the measurements for ebay.at. The site uses no keep-alive but compression. In this scenario we detect that also HSDPA and UMTS gain from the proxy system. UMTS with proxy closes up to HSDPA performance levels. The gain for EDGE and GPRS are similar to the first scenario. In numbers the gain in HSDPA and UMTS is $16 \%$ and $22 \%$ while EDGE and GPRS improve by $20 \%$ and $30 \%$.

HTTP-pipe: NO, HTTP-gzip: NO The results for the last site sms.at are shown in Fig. 4(d). This site neither supports keep-alive nor compression. In this case the proxy can add both optimizations. All technologies have a remarkable gain in this case. We want to stress the fact that HSDPAp provides identical results as ADSL. The gap between UMTS $\mathrm{u}_{p}$ and HSDPA is larger than in the results before. In this scenario with both optimizations HSDPA and UMTS gain $22 \%$ respectivly $18 \%$. EDGE and GPRS benefit even more with $28 \%$ and $44 \%$ improvement.

\subsection{Lossy compression for images}

The proxy module also features lossy image compression. The compression is available for JPG (Joint Photo- 


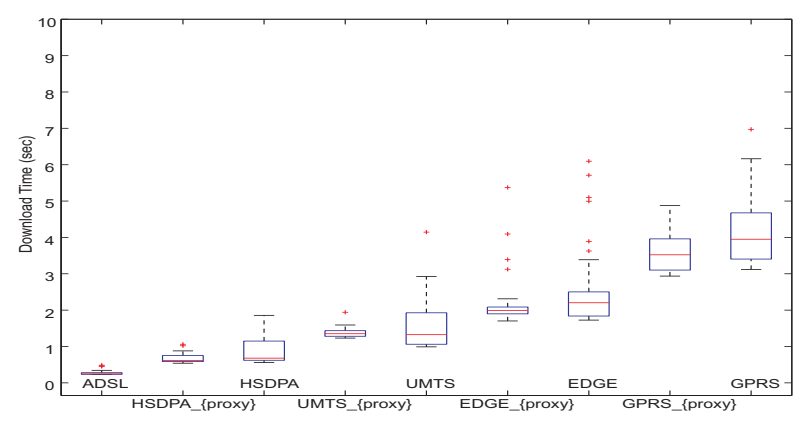

(a) google.at

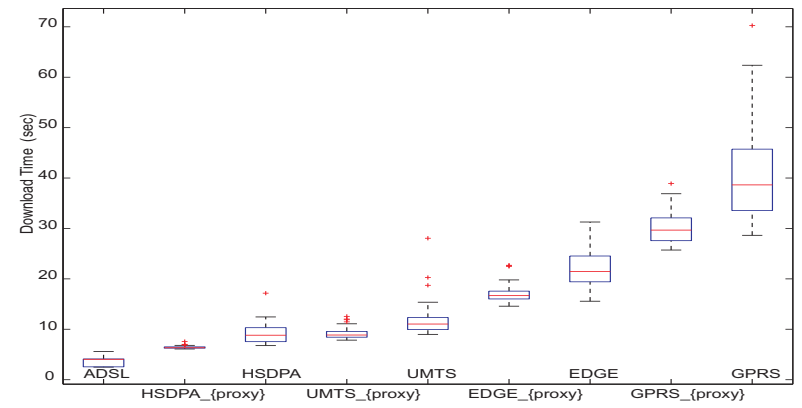

(c) ebay.at

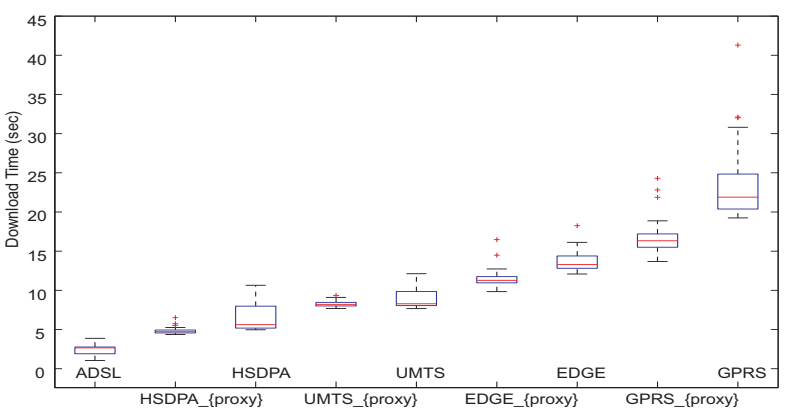

(b) orf.at

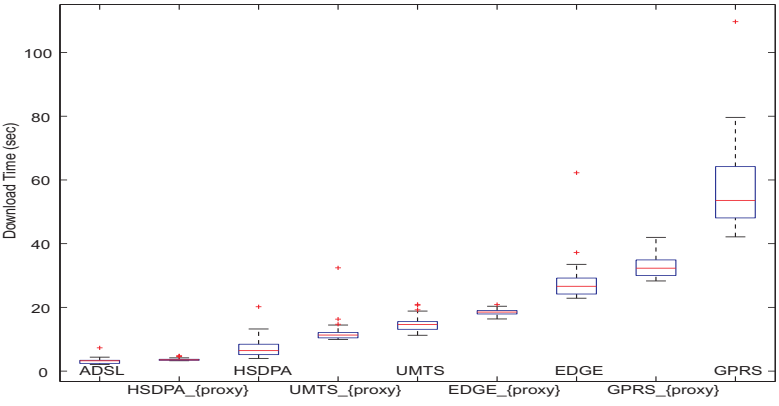

(d) sms.at

Figure 4. Technology comparison (Box-Jenkins Plot)

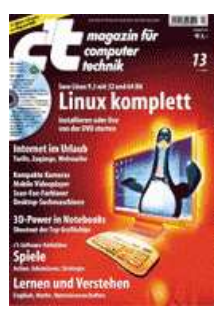

(a) Qual. $=100$

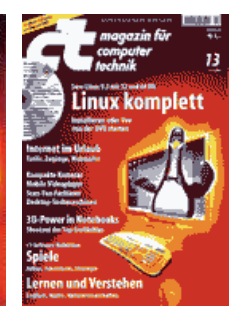

(b) Qual. $=50$

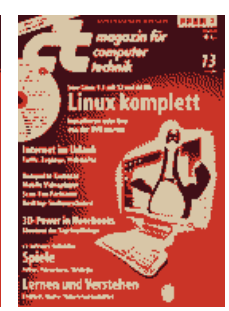

(c) Qual. $=25$

\section{Figure 6. Lossy compression of a GIF image}

graphic experts Group) and GIF (Graphics Interchange Format) images. JPG-images are delivered using a new quality factor for the quantisation. The compression rate can be manipulated by adding a special string to the requested URL (Unified Resource Locator). The number set in IctQual corresponds to the quality factor set in the JPG compression. GIF-images are basically bitmap images using a lossless compression. There is no simple way to further compress such images, only the used number of colors can be reduced. In the proxy GIF-images are compressed by reducing the used color space and apply a dithering algorithm to hide the artifacts.

The value mapping is 100 no compression, 75 good, 50 fair and 25 poor. We used this parameter in combination with the IE_control tool to retrieve several images in IctQual $=01,10,20, \ldots, 100$. The compression gain is shown in Fig. 5. Real savings in terms of volume need a set-

Table 4. FTP download time, renormalized

\begin{tabular}{|l|r|r|r|r|r|}
\hline File Size & ADSL & HSDPA & UMTS & EDGE & GPRS \\
\hline $64 \mathrm{kB}$ & 1.0 & 2.47 & 4.86 & 10.2 & 18.3 \\
$128 \mathrm{kB}$ & 1.0 & 1.93 & 3.48 & 8.33 & 15.7 \\
$256 \mathrm{kB}$ & 1.0 & 1.59 & 3.49 & 7.44 & 14.3 \\
$512 \mathrm{kB}$ & 1.0 & 1.71 & 3.44 & 7.42 & 14.7 \\
$2048 \mathrm{kB}$ & 1.0 & 1.67 & 3.54 & 7.32 & 14.5 \\
\hline
\end{tabular}

ting of below 50, which already introduces strong artifacts to the images (e.g. Fig. 6(a) to Fig. 6(c)). We assume that most of the images are already optimized to a good trade off between size and download speed. In case the compression gain is too small the proxy will deliver the original image.

\subsection{Results for FTP}

In this section we compare the results of the HTTP performance with measurements based on FTP downloads. According to the page sizes given in Table 2 we chose the following file sizes for the test: $64 \mathrm{kB}, 256 \mathrm{kB}, 512 \mathrm{kB}$ and $2048 \mathrm{kB}$. The following Table 4 shows the FTP performance index or relative download time for all technologies and file sizes. The numbers in the table are normalized to the ADSL values and averaged over ten measurements. In the $64 \mathrm{kB}$ case the factors between the technologies are similar to the results obtained in the HTTP non-proxy case. However, in the $2048 \mathrm{kB}$ case the factors are similar to the proxy case in Table 3. The main change in download performance is achieved between $64 \mathrm{kB}$ and $256 \mathrm{kB}$. This result also corresponds to the way the proxy changes the HTTP transfers. In 
the optimized case all objects are piped into a single connection, therefore we obtain the FTP performance of approximately $512 \mathrm{kB}$ (compare page size of the sample shown in Table 2). In case of no optimization the web page consists of many small objects and the performance will be diminished.

\section{Related Work}

There are several books covering the performance of mobile Internet access technologies [1,5]. However, common literature $[6,7]$ uses TCP bulk transfers (e.g. FTP) to evaluate network performance. As we have shown in the section concerning FTP download times, due to the high RTT and drop rates, the results strongly depend on the file sizes. The following papers $[8,9]$ report about TCP in high delay environments, but with no link to mobile Internet accesses. We found one paper measuring HTTP performance in GPRS networks [10]. The paper reports connection keep-alive as the main improvement in the scenario. R. Chakravoty et al. presents in [11] a detailed overview of the impact of various optimizations to the download time of HTTP pages for dialup, GPRS and UMTS. However to our knowledge, there is no work comparing GPRS, EDGE, UMTS and HSDPA using HTTP measurements up to now.

\section{Summary and Conclusions}

In this paper we evaluated the HTTP-performance of different mobile Internet access technologies, namely GPRS, EDGE, UMTS and HSDPA. The results point out that HSDPA is a good solution for mobile web users nearly reaching the performance of ADSL. It achieves a speed up factor of two over UMTS. The benchmarks for EDGE reveal an ambivalent result. If the proxy system is used a performance close to UMTS is achieved. However, without the proxy this technology suffers from the system inherent high delays and drops to cached GPRS performance numbers. In the non proxy case GPRS on average needs 16.8 times the ADSL download time, which is a very poor performance. We can conclude that the impact of the proxy is linked to the bandwidth delay product. As seen in EDGE a high delay and bandwidth scenario will profit most. The detailed analysis of the proxy versus non-proxy results reveals that the main improvement of the proxy system is connection keep-alive $(\approx 15-30 \%)$. If compression is applied, mainly GPRS and EDGE benefit from the reduced transfer volume $(\approx 5-25 \%)$.

We also compared various settings of lossy compression. The results showed that most of the images tested were already optimized to some extend. The test set already used a JPG quality factor of $\leq 60$ by default, further compressions led to strong artifacts within the images.

In the last paragraph we investigated the difference between using FTP for performance evaluation and our per- formance index. The FTP measurements delivered results similar to HTTP, depending on the chosen file sizes. Small file sizes $(64 k B)$ led to results similar to the non proxy case, while files with sizes of $\geq 256 \mathrm{kB}$ performed according to the HTTP results using a proxy.

The lesson learned from this work is that mobile operators should, at least, apply such a proxy system to their $2.5 \mathrm{G}$ access as it delivers a strong gain there. It will be interesting to see how technologies like WiMax, which have a higher bandwidth than HSDPA but the same delay, will perform for web services.

Our next step in research is the setup of a long term recording system in an operator's network and the extraction of the parameters from the traces. Parallel to this the development of a performance metric is ongoing.

\section{Acknowledgments}

Special thanks go to Markus Hogl who assisted us with his Visual C++ knowledge in coding the tool. The paper is part of the DARWIN project. This project was partly supported by the Austrian K-Plus. The views expressed in this paper are those of the authors and do not necessarily reflect the views of the project partners.

\section{References}

[1] M. Taferner and E. Bonek. Wireless internet access over GSM and UMTS. Springer, 2002.

[2] P. Svoboda and F. Ricciato. "Composition of GPRS and UMTS traffic: snapshots from a live network". In IPS MoMe 2006, Salzburg, pages 42-44, February 2006.

[3] OneStat. Mozilla's browsers global usage share is still growing. Technical report, OneStat, 2006.

[4] TS 124008. Mobile radio interface Layer 3 specification; Core network protocols; Stage 3. ETSI/3GPP.

[5] T. Halonen, J. Romero, and J. Melero. GSM, GPRS and EDGE Performance: Evolution Towards 3G/UMTS. WILEY, 2003.

[6] Y. Lee. "Measured TCP Performance in CDMA 1x EV-DO Networks". In PAM 2005, pages 208-221, 2005.

[7] K. Pentikousis, M. Palola, and M. Jurvansuu. "Active Goodput Measurements from a Public 3G/UMTS Network". In Communications Letters IEEE, pages 802- 804, Jun 2005.

[8] T. Lakshman and U. Madhow. "The performance of TCP/IP for networks with high bandwidth-delay products and random loss". IEEE/ACM Transactions on Networking, 5(3):336-350, July 1997.

[9] W. Zhao, T. Seth, M. Kim, and M. Willebeek-LeMair. "Optimal Bandwidth/Delay Tradeoff for Feasible-Region-Based Scalabel Multimedia Scheduling”. In INFOCOM (3), pages 1131-1138, 1998.

[10] R. Chakrvorty and I. Pratt. "WWW performance over GPRS". In Mobile and Wireless Communications Network. 4th International Workshop, pages 527-531, Sep 2002.

[11] R. Chakravorty, S. Banerjee, P. Rodriguez, J. Chesterfield, and I. Pratt. Performance optimizations for wireless wide-area networks: comparative study and experimental evaluation. In MobiCom '04, pages 159-173, New York, NY, USA, 2004. ACM Press. 